\title{
Quantitative self adjoint operator direct approximations
}

\author{
George A. Anastassiou \\ Department of Mathematical Sciences, University of Memphis, Memphis, TN 38152, U.S.A.
}

Communicated by $R$. Saadati

\begin{abstract}
Here we give a series of self adjoint operator positive linear operators general results. Then we present specific similar results related to neural networks. This is a quantitative treatment to determine the degree of self adjoint operator uniform approximation with rates, of sequences of self adjoint positive linear operators in general, and in particular of self adjoint specific neural network operators. The approach is direct relying on Gelfand's isometry. (c)2017 All rights reserved.
\end{abstract}

Keywords: Self adjoint operator, Hilbert space, positive linear operator, Bernstein polynomials, neural network operators. 2010 MSC: 41A17, 41A25, 41A36, 41A80, 47A58, 47A60, 47A67.

\section{Background}

Let $A$ be a self adjoint linear operator on a complex Hilbert space $(H ;\langle\cdot, \cdot\rangle)$. The Gelfand map establishes a $*$-isometrically isomorphism $\Phi$ between the set $\mathrm{C}(\mathrm{Sp}(A))$ of all continuous functions defined on the spectrum of $A$, denoted by $\operatorname{Sp}(A)$, and the $C^{*}$-algebra $C^{*}(A)$ generated by $A$ and the identity operator $1_{\mathrm{H}}$ on $\mathrm{H}$ as follows (see e.g. [9, p. 3]):

For any $f, g \in C(\operatorname{Sp}(A))$ and any $\alpha, \beta \in \mathbb{C}$ we have

(i) $\Phi(\alpha f+\beta g)=\alpha \Phi(f)+\beta \Phi(g)$;

(ii) $\Phi(\mathrm{fg})=\Phi(\mathrm{f}) \Phi(\mathrm{g})$ (the operation composition is on the right) and $\Phi(\overline{\mathrm{f}})=(\Phi(\mathrm{f}))^{*}$;

(iii) $\|\Phi(f)\|=\|f\|:=\sup _{t \in \operatorname{Sp}(A)}|f(t)|$;

(iv) $\Phi\left(f_{0}\right)=1_{H}$ and $\Phi\left(f_{1}\right)=A$, where $f_{0}(t)=1$ and $f_{1}(t)=t$, for $t \in S p(A)$.

With this notation we define

$$
f(A):=\Phi(f), \quad \text { for all } f \in C(\operatorname{Sp}(A)),
$$

and we call it the continuous functional calculus for a self adjoint operator $A$.

If $A$ is a self adjoint operator and $f$ is a real valued continuous function on $\operatorname{Sp}(A)$ then $f(t) \geqslant 0$ for any $t \in S p(A)$ implies that $f(A) \geqslant 0$, i.e. $f(A)$ is a positive operator on $H$. Moreover, if both $f$ and $g$ are real-valued continuous functions on $\operatorname{Sp}(A)$ then the following important property holds:

Email address: ganastss@memphis .edu (George A. Anastassiou)

doi:10.22436/jnsa.010.05.45 
(P) $f(t) \geqslant g(t)$ for any $t \in S p(A)$, implies that $f(A) \geqslant g(A)$ in the operator order of $B(H)$.

Equivalently, we use (see [6, pp. 7-8]):

Let $U$ be a self adjoint operator on the complex Hilbert space $(H,\langle\cdot, \cdot\rangle)$ with the spectrum $S p(U)$ included in the interval $[m, M]$ for some real numbers $m<M$ and $\left\{E_{\lambda}\right\}_{\lambda}$ be its spectral family.

Then for any continuous function $f:[a, b] \rightarrow \mathbb{C}$, where $[m, M] \subset(a, b)$, it is well-known that we have the following spectral representation in terms of the Riemann-Stieljes integral:

$$
\langle f(U) x, y\rangle=\int_{m-0}^{M} f(\lambda) d\left(\left\langle E_{\lambda} x, y\right\rangle\right)
$$

for any $x, y \in H$. The function $g_{x, y}(\lambda):=\left\langle E_{\lambda} x, y\right\rangle$ is of bounded variation on the interval $[m, M]$, and

$$
g_{x, y}(m-0)=0, \quad \text { and } \quad g_{x, y}(M)=\langle x, y\rangle,
$$

for any $x, y \in H$. Furthermore, it is known that $g_{x}(\lambda):=\left\langle E_{\lambda} x, x\right\rangle$ is increasing and right continuous on $[\mathrm{m}, \mathrm{M}]$.

In this article we will use a lot the formula

$$
\langle f(U) x, x\rangle=\int_{m-0}^{M} f(\lambda) d\left(\left\langle E_{\lambda} x, x\right\rangle\right), \quad \forall x \in H .
$$

As a symbol we can write

$$
f(U)=\int_{m-0}^{M} f(\lambda) d E_{\lambda}
$$

Above, $\mathrm{m}=\min \{\lambda \mid \lambda \in \mathrm{Sp}(\mathrm{U})\}:=\min \mathrm{Sp}(\mathrm{U}), M=\max \{\lambda \mid \lambda \in \mathrm{Sp}(\mathrm{U})\}:=\max \mathrm{Sp}(\mathrm{U})$. The projections $\left\{E_{\lambda}\right\}_{\lambda \in \mathbb{R}}$, are called the spectral family of $A$, with the properties:

(a) $E_{\lambda} \leqslant E_{\lambda^{\prime}}$ for $\lambda \leqslant \lambda^{\prime}$;

(b) $E_{m-0}=0_{H}$ (zero operator), $E_{M}=1_{H}$ (identity operator) and $E_{\lambda+0}=E_{\lambda}$ for all $\lambda \in \mathbb{R}$.

Furthermore

$$
E_{\lambda}:=\varphi_{\lambda}(\mathrm{U}), \quad \forall \lambda \in \mathbb{R},
$$

is a projection which reduces $\mathrm{U}$, with

$$
\varphi_{\lambda}(s):= \begin{cases}1, & \text { for }-\infty<s \leqslant \lambda, \\ 0, & \text { for } \lambda<s<+\infty .\end{cases}
$$

The spectral family $\left\{E_{\lambda}\right\}_{\lambda \in \mathbb{R}}$ determines uniquely the self-adjoint operator $U$ and vice versa.

For more on the topic see [7, pp. 256-266], and for more details see there pp. 157-266. See also [5].

Some more basics are given in ([6, pp. 1-5]):

Let $(H ;\langle\cdot, \cdot\rangle)$ be a Hilbert space over $\mathbb{C}$. A bounded linear operator $A$ defined on $H$ is selfjoint, i.e., $A=A^{*}$, iff $\langle A x, x\rangle \in \mathbb{R}$, for all $x \in H$, and if $A$ is self adjoint, then

$$
\|A\|=\sup _{x \in H:\|x\|=1}|\langle A x, x\rangle| .
$$

Let $A$, $B$ be self adjoint operators on $H$. Then $A \leqslant B$, iff $\langle A x, x\rangle \leqslant\langle B x, x\rangle$, for all $x \in H$.

In particular, $A$ is called positive if $A \geqslant 0$.

Denote by

$$
\mathcal{P}:=\left\{\varphi(s):=\sum_{k=0}^{n} \alpha_{k} s^{k} \mid n \geqslant 0, \alpha_{k} \in \mathbb{C}, 0 \leqslant k \leqslant n\right\} .
$$


If $A \in \mathcal{B}(H)$ (the Banach algebra of all bounded linear operators defined on $H$, i.e., from $H$ into itself) is self adjoint, and $\varphi(s) \in \mathcal{P}$ has real coefficients, then $\varphi(A)$ is self adjoint, and

$$
\|\varphi(A)\|=\max \{|\varphi(\lambda)|, \lambda \in \operatorname{Sp}(A)\} .
$$

If $\varphi$ is any function defined on $\mathbb{R}$ we define

$$
\|\varphi\|_{A}:=\sup \{|\varphi(\lambda)|, \lambda \in \operatorname{Sp}(A)\} .
$$

If $A$ is self adjoint operator on Hilbert space $H$ and $\varphi$ is continuous and given that $\varphi(A)$ is self adjoint, then $\|\varphi(A)\|=\|\varphi\|_{A}$. And if $\varphi$ is a continuous real-valued function, so it is $|\varphi|$, then $\varphi(A)$ and $|\varphi|(A)=|\varphi(A)|$ are self adjoint operators (by [6, p. 4, Theorem 7]).

Hence it holds

$$
\begin{aligned}
\||\varphi(A)|\| & =\|\varphi \mid\|_{A}=\sup \{\|\varphi(\lambda)\|, \lambda \in \operatorname{Sp}(A)\} \\
& =\sup \{|\varphi(\lambda)|, \lambda \in \operatorname{Sp}(A)\}=\|\varphi\|_{A}=\|\varphi(A)\|,
\end{aligned}
$$

that is,

$$
\|\varphi(A) \mid\|=\|\varphi(A)\| .
$$

For a self adjoint operator $A \in \mathcal{B}(H)$ which is positive, there exists a unique positive self adjoint operator $B:=\sqrt{A} \in \mathcal{B}(H)$ such that $B^{2}=A$, that is $(\sqrt{A})^{2}=A$. We call $B$ the square root of $A$.

Let $A \in \mathcal{B}(H)$, then $A^{*} A$ is self adjoint and positive. Define the "operator absolute value" $|A|:=\sqrt{A^{*} A}$. If $A=A^{*}$, then $|A|=\sqrt{A^{2}}$.

For a continuous real-valued function $\varphi$ we observe the following:

$$
\begin{aligned}
|\varphi(A)| \text { (the functional absolute value) } & =\int_{m-0}^{M}|\varphi(\lambda)| \mathrm{dE}_{\lambda} \\
& =\int_{\mathfrak{m}-0}^{M} \sqrt{(\varphi(\lambda))^{2}} \mathrm{dE}_{\lambda} \\
& =\sqrt{(\varphi(A))^{2}}=|\varphi(\mathrm{A})| \text { (operator absolute value), }
\end{aligned}
$$

where $A$ is a self adjoint operator.

That is we have

$$
|\varphi(A)| \text { (functional absolute value) }=|\varphi(A)| \text { (operator absolute value). }
$$

The next comes from [5, p. 3]:

We say that a sequence $\left\{A_{n}\right\}_{n=1}^{\infty} \subset \mathcal{B}(H)$ converges uniformly to $A$ (convergence in norm), iff

$$
\lim _{n \rightarrow \infty}\left\|A_{n}-A\right\|=0
$$

and $w$ denote it as $\lim _{n \rightarrow \infty} A_{n}=A$.

We will use Hölder's-McCarthy inequality ([8]): Let $A$ be a self adjoint positive operator on a Hilbert space $H$. Then

$$
\left\langle A^{r} x, x\right\rangle \leqslant\langle A x, x\rangle^{r}
$$

for all $0<\mathrm{r}<1$ and $x \in \mathrm{H}:\|x\|=1$.

Let $A, B \in \mathcal{B}(H)$, then

$$
\|A B\| \leqslant\|A\|\|B\|
$$

by Banach algebra property. 


\section{Main results}

Our approach is direct based on Gelfand's isometry.

All the functions we are dealing here are real-valued. We assume that $S p(A) \subseteq[m, M]$.

Let $\left\{L_{n}\right\}_{\mathfrak{n} \in \mathbb{N}}$ be a sequence of positive linear operators from $C([m, M])$ into itself (i.e., if $f, g \in$ $C([m, M])$ such that $f \geqslant g$, then $\left.L_{n}(f) \geqslant L_{n}(g)\right)$. It is interesting to study the convergence of $L_{n} \rightarrow I$ (unit operator, i.e., $I(f)=f$, for all $f \in C([m, M]))$. By property (i) we have that

$$
\Phi\left(L_{n} f-f\right)=\Phi\left(L_{n} f\right)-\Phi(f)=\left(L_{n} f\right)(A)-f(A),
$$

and

$$
\Phi\left(\mathrm{L}_{n} 1 \pm 1\right)=\Phi\left(\mathrm{L}_{n} 1\right) \pm \Phi(1)=\left(\mathrm{L}_{n} 1\right)(\mathrm{A}) \pm 1_{\mathrm{H}},
$$

the last comes by property (iv).

And by property (iii) we obtain

$$
\left\|\Phi\left(L_{n} f-f\right)\right\|=\left\|\left(L_{n} f\right)(A)-f(A)\right\|=\left\|L_{n} f-f\right\|,
$$

and

$$
\left\|\Phi\left(\mathrm{L}_{n} 1 \pm 1\right)\right\|=\left\|\left(\mathrm{L}_{n} 1\right)(A) \pm 1_{\mathrm{H}}\right\|=\left\|\mathrm{L}_{n}(1) \pm 1\right\| .
$$

We need the first modulus of continuity

$$
\omega_{1}(f, \delta):=\sup _{\substack{x, y \in[m, M] \\|x-y| \leqslant \delta}}|f(x)-f(y)|, \quad \delta>0,
$$

here $\|\cdot\|_{\infty}$ stands for the sup-norm over $[m, M]$.

We need and mention:

Theorem 2.1 ([1, p. 419]). Consider the positive linear operator

$$
\mathrm{L}: \mathrm{C}([\mathrm{m}, \mathrm{M}]) \rightarrow \mathrm{C}([\mathrm{m}, \mathrm{M}]) .
$$

Define $(\mathrm{n} \in \mathbb{N})$

$$
\mathrm{D}_{\mathrm{n}}:=\left\|\left(\mathrm{L}\left(|\mathrm{t}-\cdot|^{\mathrm{n}}\right)\right)(\cdot)\right\|_{\infty}^{\frac{1}{n}} .
$$

Let $\mathrm{f} \in \mathrm{C}^{\mathrm{n}}([\mathrm{m}, \mathrm{M}])$. Then

$$
\begin{aligned}
\|L f-f\|_{\infty} \leqslant & \|f\|_{\infty} \cdot\|L 1-1\|_{\infty}+\sum_{k=1}^{n} \frac{\left\|f^{(k)}\right\|_{\infty}}{k !}\left\|\left(L(t-\cdot)^{k}\right)(\cdot)\right\|_{\infty} \\
& +\omega_{1}\left(f^{(n)}, D_{n}\right) \cdot D_{n}^{n-1}\left(\frac{(M-m)}{(n+1) !}+\frac{D_{n}}{2 n !}+\frac{D_{n}^{2}}{8(M-m)(n-1) !}\right) .
\end{aligned}
$$

By [1, p. 415], we have $\mathrm{D}_{\mathrm{n}}<+\infty$.

We derive:

Theorem 2.2. Let all as in Theorem 2.1 hold. Then

$$
\begin{aligned}
\|(\operatorname{Lf})(A)-f(A)\| \leqslant & \|f(A)\|\left\|(\operatorname{L1})(A)-1_{H}\right\| \\
& +\sum_{k=1}^{n} \frac{\left\|f^{(k)}(A)\right\|}{k !}\left\|\left(L(t-A)^{k}\right)(A)\right\| \\
& +\omega_{1}\left(f^{(n)}, D_{n}\right) D_{n}^{n-1}\left(\frac{(M-m)}{(n+1) !}+\frac{D_{n}}{2 n !}+\frac{D_{n}^{2}}{8(M-m)(n-1) !}\right),
\end{aligned}
$$

where

$$
D_{n}=\left\|\left(L\left(|t-A|^{n}\right)\right)(A)\right\|^{\frac{1}{n}} .
$$


We mention:

Corollary 2.3 ([1, p. 421]). Let $\mathrm{L}$ be a positive linear operator from $\mathrm{C}([\mathrm{m}, \mathrm{M}])$ into itself. Here

$$
\mathrm{D}_{1}:=\|(\mathrm{L}(|\mathrm{t}-\cdot|))(\cdot)\|_{\infty}<+\infty .
$$

Let $\mathrm{f} \in \mathrm{C}^{1}([\mathrm{~m}, \mathrm{M}])$. Then

$$
\begin{aligned}
\|\mathrm{Lf}-\mathrm{f}\|_{\infty} \leqslant & \|f\|_{\infty} \cdot\|\mathrm{L} 1-1\|_{\infty}+\left\|\mathrm{f}^{\prime}\right\|_{\infty}\|(\mathrm{L}(\mathrm{t}-\cdot))(\cdot)\|_{\infty} \\
& +\frac{1}{2} \omega_{1}\left(\mathrm{f}^{\prime}, \mathrm{D}_{1}\right)\left((M-\mathrm{m})+\mathrm{D}_{1}+\frac{\mathrm{D}_{1}^{2}}{4(\mathrm{M}-\mathrm{m})}\right) .
\end{aligned}
$$

We obtain:

Corollary 2.4. Let all as in Corollary 2.3 hold. Then

$$
\begin{aligned}
\|(\operatorname{Lf})(A)-f(A)\| \leqslant & \|f(A)\|\left\|(L 1)(A)-1_{H}\right\|+\left\|f^{\prime}(A)\right\|\|(L(t-A))(A)\| \\
& +\frac{1}{2} \omega_{1}\left(f^{\prime}, D_{1}\right)\left((M-m)+D_{1}+\frac{D_{1}^{2}}{4(M-m)}\right)
\end{aligned}
$$

where

$$
D_{1}=\|(L(|t-A|))(A)\| .
$$

We mention:

Corollary 2.5 ([1, p. 421]). Let L be a positive linear operator from $\mathrm{C}([\mathrm{m}, \mathrm{M}])$ into itself. Here

$$
\mathrm{D}_{2}:=\left\|\left(\mathrm{L}\left((\mathrm{t}-\cdot)^{2}\right)\right)(\cdot)\right\|_{\infty}^{\frac{1}{2}}<+\infty .
$$

Let $\mathrm{f} \in \mathrm{C}^{2}([\mathrm{~m}, \mathrm{M}])$. Then

$$
\begin{aligned}
\|L f-f\|_{\infty} \leqslant & \|f\|_{\infty} \cdot\|L 1-1\|_{\infty}+\left\|f^{\prime}\right\|_{\infty}\|(L(t-\cdot))(\cdot)\|_{\infty} \\
& +\frac{\left\|f^{\prime \prime}\right\|_{\infty}}{2}\left\|\left(L(t-\cdot)^{2}\right)(\cdot)\right\|_{\infty} \\
& +\frac{1}{2} \omega_{1}\left(f^{\prime \prime}, D_{2}\right) \cdot D_{2}\left(\frac{(M-m)}{3}+\frac{D_{2}}{2}+\frac{D_{2}^{2}}{4(M-m)}\right) .
\end{aligned}
$$

We derive:

Corollary 2.6. Let all as in Corollary 2.5 hold. Then

$$
\begin{aligned}
\|(\operatorname{Lf})(A)-f(A)\| \leqslant & \|f(A)\|\left\|(L 1)(A)-1_{H}\right\|+\left\|f^{\prime}(A)\right\|\|(L(t-A))(A)\|_{\infty} \\
& +\frac{\left\|f^{\prime \prime}(A)\right\|}{2}\left\|\left(L(t-A)^{2}\right)(A)\right\|_{\infty} \\
& +\frac{1}{2} \omega_{1}\left(f^{\prime \prime}, D_{2}\right) D_{2}\left(\frac{(M-m)}{3}+\frac{D_{2}}{2}+\frac{D_{2}^{2}}{4(M-m)}\right),
\end{aligned}
$$

where

$$
\mathrm{D}_{2}=\left\|\left(\mathrm{L}\left((\mathrm{t}-\mathrm{A})^{2}\right)\right)(\mathrm{A})\right\|^{\frac{1}{2}}
$$

We give: 
Example 2.7. Let $f \in C([0,1])$, the basic Bernestein polynomial operators are defined by

$$
\left(\beta_{n}(f)\right)(t):=\sum_{k=0}^{n} f\left(\frac{k}{n}\right)\left(\begin{array}{l}
n \\
k
\end{array}\right) t^{k}(1-t)^{n-k}, \quad t \in[0,1] .
$$

By $[1$, p. 421$]$, when $f \in C^{2}([0,1])$ we get

$$
\left\|\beta_{n}(f)-f\right\|_{\infty} \leqslant \frac{\left\|f^{\prime \prime}\right\|_{\infty}}{8 n}+\frac{1}{4 \sqrt{n}} \omega_{1}\left(f^{\prime \prime}, \frac{1}{2 \sqrt{n}}\right)\left(\frac{1}{3}+\frac{1}{4 \sqrt{n}}+\frac{1}{16 n}\right) .
$$

The map

$$
[\mathrm{m}, M] \ni s=\varphi(t)=(M-m) t+m, \quad t \in[0,1],
$$

maps $1-1$ and onto : $[0,1]$ onto $[m, M]$.

Let now $f \in C^{2}([m, M])$, then

$$
f(s)=f(\varphi(t))=f((M-m) t+m),
$$

and

Furthermore it holds

$$
\frac{d f(s)}{d t}=(f(\varphi(t)))^{\prime}=f^{\prime}(\varphi(t))(M-m)=f^{\prime}(s)(M-m) .
$$

We observe that $(t \in[0,1])$

$$
\frac{d^{2} f(s)}{d t^{2}}=f^{\prime \prime}(s)(M-m)^{2}
$$

$$
\begin{aligned}
\left(\beta_{n}(f((M-m) t+m))\right)(t) & =\sum_{k=0}^{n}\left(f\left((M-m) \frac{k}{n}+m\right)\right)\left(\begin{array}{l}
n \\
k
\end{array}\right) t^{k}(1-t)^{n-k} \\
& =\sum_{k=0}^{n}\left(f\left((M-m) \frac{k}{n}+m\right)\right)\left(\begin{array}{l}
n \\
k
\end{array}\right)\left(\frac{s-m}{M-m}\right)^{k}\left(\frac{M-s}{M-m}\right)^{n-k} \\
& =:\left(B_{n}(f)\right)(s), \quad s \in[m, M] .
\end{aligned}
$$

The operators $\left(B_{n}(f)\right)(s)$ are the general Bernstein polynomials. As in [4], we get that

$$
\omega_{1}(f((M-m) t+m), \delta)=\omega_{1}(f,(M-m) \delta),
$$

where $f \in C([m, M])$.

Here the function $f((M-m) t+m) \in C([0,1])$, as a function of $t \in[0,1]$.

So we apply (2.4), for $f((M-m) t+m), t \in[0,1]$, we obtain

$$
\left\|B_{n}(f)-f\right\|_{\infty} \leqslant(M-m)^{2}\left[\frac{\left\|f^{\prime \prime}\right\|_{\infty}}{8 n}+\frac{1}{4 \sqrt{n}} \omega_{1}\left(f^{\prime \prime}, \frac{(M-m)}{2 \sqrt{n}}\right)\left(\frac{1}{3}+\frac{1}{4 \sqrt{n}}+\frac{1}{16 n}\right)\right],
$$

where $f \in C([m, M])$.

Consequently, we obtain

$$
\left\|\left(B_{n}(f)\right)(A)-f(A)\right\| \leqslant(M-m)^{2}\left[\frac{\left\|f^{\prime \prime}(A)\right\|}{8 n}+\frac{1}{4 \sqrt{n}} \omega_{1}\left(f^{\prime \prime}, \frac{(M-m)}{2 \sqrt{n}}\right)\left(\frac{1}{3}+\frac{1}{4 \sqrt{n}}+\frac{1}{16 n}\right)\right],
$$

for all $f \in C([m, M])$.

We need:

Theorem 2.8 ([1, p. 422]). Let $\mathrm{L} \neq 0$ be a positive linear operator from $\mathrm{C}([\mathrm{m}, \mathrm{M}])$ into itself. Set

$$
\rho:=\left\|\left(\mathrm{L}(\mathrm{t}-\mathrm{x})^{2}\right)(\mathrm{x})\right\|_{\infty}^{\frac{1}{2}}
$$

and consider $r>0$. Let $f \in \mathrm{C}^{1}([\mathrm{~m}, \mathrm{M}])$. Then 


$$
\begin{aligned}
\|L f-f\|_{\infty} & -\|f\|_{\infty}\|L 1-1\|_{\infty}-\left\|f^{\prime}\right\|_{\infty}\|(L(t-x))(x)\|_{\infty} \\
& \leqslant\left\{\begin{array}{l}
\frac{1}{8 r}\left(2+\sqrt{\|L(1)\|_{\infty}} r\right)^{2} \omega_{1}\left(f^{\prime}, r \rho\right) \rho, \quad \text { if } r \leqslant \frac{2}{\sqrt{\|L(1)\|_{\infty}}}, \\
\sqrt{\|L(1)\|_{\infty}} \omega_{1}\left(f^{\prime}, r \rho\right) \rho, \quad \text { if } r>\frac{2}{\sqrt{\|L(1)\|_{\infty}}},
\end{array}\right.
\end{aligned}
$$

by [1, p. 415], we have that $\rho<+\infty$.

An improved results for $f \in C^{1}([m, M])$ follows:

Theorem 2.9. Let all as in Theorem 2.8 hold. Then

$$
\begin{aligned}
\|(L f)(A) & -f(A)\|-\| f(A)\|\|(L 1)(A)-1_{H}\|-\| f^{\prime}(A)\|\|(L(t-A))(A) \| \\
& \leqslant\left\{\begin{array}{l}
\frac{1}{8 r}(2+\sqrt{\|(L(1))(A)\| r})^{2} \omega_{1}\left(f^{\prime}, r \rho\right) \rho, \quad \text { if } r \leqslant \frac{2}{\sqrt{\|(L(1))(A)\|}}, \\
\sqrt{\|(L(1))(A)\|} \omega_{1}\left(f^{\prime}, r \rho\right) \rho, \quad \text { if } r>\frac{2}{\sqrt{\|(L(1))(A)\|}},
\end{array}\right.
\end{aligned}
$$

where

$$
\rho=\left\|\left(L(t-A)^{2}\right)(A)\right\|^{\frac{1}{2}} .
$$

We continue with neural network operators.

Definition 2.10 (see [2, pp. 3-12]). We consider here the sigmoidal function of logarithmic type

$$
s(x)=\frac{1}{1+e^{-x}}, \quad x \in \mathbb{R},
$$

and

$$
\Phi(x)=\frac{1}{2}(s(x+1)-s(x-1))>0, \quad \forall x \in \mathbb{R} .
$$

Let $f \in C([m, M])$ and $n \in \mathbb{N}$, such that $\lceil n m\rceil \leqslant\lfloor n M\rfloor(\lceil\cdot\rceil$ is the ceiling and $\lfloor\cdot\rfloor$ is the integral part of the number).

We consider the positive linear neural network operator

$$
G_{n}(f, x)=\frac{\sum_{k=\lceil n m\rceil}^{\lfloor n M\rfloor} f\left(\frac{k}{n}\right) \Phi(n x-k)}{\sum_{k=\lceil n m\rceil}^{\lfloor n M\rfloor} \Phi(n x-k)}, \quad x \in[m, M]
$$

Clearly, $G_{n}: C([m, M]) \hookrightarrow C([m, M])$. For large enough $n$ we always have $\lceil n m\rceil \leqslant\lfloor n M\rfloor$. Also $m \leqslant \frac{k}{n} \leqslant$ $M$, iff $\lceil n m\rceil \leqslant k \leqslant\lfloor n M\rfloor$.

We need and mention:

Theorem 2.11 (see $[2$, p. 9]). Let $\mathrm{f} \in \mathrm{C}([\mathrm{m}, \mathrm{M}]), 0<\alpha<1$. Then

$$
\left\|G_{n}(f)-f\right\|_{\infty} \leqslant(5.250312578)\left[\omega_{1}\left(f, \frac{1}{n^{\alpha}}\right)+6.3984\|f\|_{\infty} e^{-n^{(1-\alpha)}}\right] .
$$

We derive:

Theorem 2.12. Let $\mathrm{f} \in \mathrm{C}([\mathrm{m}, \mathrm{M}]), 0<\alpha<1$. Then

$$
\left\|\left(G_{n}(f)\right)(A)-f(A)\right\| \leqslant(5.250312578)\left[\omega_{1}\left(f, \frac{1}{n^{\alpha}}\right)+6.3984\|f(A)\| e^{-n^{(1-\alpha)}}\right] .
$$


We mention:

Theorem 2.13 ([2, p. 11]). Let $f \in C^{N}([m, M]), N \in \mathbb{N}, 0<\alpha<1$. Then

$$
\begin{aligned}
\left\|G_{n}(f)-f\right\|_{\infty} \leqslant & (5.250312578) \\
& \times\left\{\sum_{j=1}^{N} \frac{\left\|f^{(j)}\right\|_{\infty}}{j !}\left[\frac{1}{n^{\alpha j}}+(3.1992)(M-m)^{j} e^{-n^{(1-\alpha)}}\right]\right. \\
& \left.+\left[\omega_{1}\left(f^{(N)}, \frac{1}{n^{\alpha}}\right) \frac{1}{n^{\alpha N} N !}+(6.3984) \frac{\left\|f^{(N)}\right\|_{\infty}}{N !}(M-m)^{N} e^{-n^{(1-\alpha)}}\right]\right\} .
\end{aligned}
$$

To obtain:

Theorem 2.14. Let $\mathrm{f} \in \mathrm{C}^{\mathrm{N}}([\mathrm{m}, \mathrm{M}]), \mathrm{N} \in \mathbb{N}, 0<\alpha<1$. Then

$$
\begin{aligned}
\left\|\left(G_{n}(f)\right)(A)-f(A)\right\| \leqslant & (5.250312578) \\
& \times\left\{\sum_{j=1}^{N} \frac{\left\|f^{(j)}(A)\right\|}{j !}\left[\frac{1}{n^{\alpha j}}+(3.1992)(M-m)^{j} e^{-n^{(1-\alpha)}}\right]\right. \\
& +\left[\omega_{1}\left(f^{(N)}, \frac{1}{n^{\alpha}}\right) \frac{1}{n^{\alpha N} N !}+(6.3984) \frac{\left\|f^{(N)}(A)\right\|}{N !}(M-m)^{N} e^{\left.\left.-n^{(1-\alpha)}\right]\right\} .}\right.
\end{aligned}
$$

We need:

Definition 2.15 ([2, pp. 34-45]). We consider the hyperbolic tangent function $\tanh x, x \in \mathbb{R}$ :

$$
\tanh x:=\frac{e^{x}-e^{-x}}{e^{x}+e^{-x}}
$$

and

$$
\Psi(x):=\frac{1}{4}(\tanh (x+1)-\tanh (x-1))>0,
$$

for all $x \in \mathbb{R}$.

Let $f \in C([m, M])$ and $n \in \mathbb{N}:\lceil n m\rceil \leqslant\lfloor n M\rfloor$. We consider the positive linear neural network operator

$$
F_{n}(f, x)=\frac{\sum_{k=\lceil n m\rceil}^{\lfloor n M\rfloor} f\left(\frac{k}{n}\right) \Psi(n x-k)}{\sum_{k=\lceil n m\rceil}^{\lfloor n M\rfloor} \Psi(n x-k)}, \quad x \in[m, M] .
$$

Clearly, $F_{n}: C([m, M]) \hookrightarrow C([m, M])$.

We mention:

Theorem 2.16 ([2, p. 42]). Let $\mathrm{f} \in \mathrm{C}([\mathrm{m}, \mathrm{M}]), 0<\alpha<1$. Then

$$
\left\|F_{n}(f)-f\right\|_{\infty} \leqslant(4.1488766)\left[\omega_{1}\left(f, \frac{1}{n^{\alpha}}\right)+2 e^{4}\|f\|_{\infty} e^{-2 n^{(1-\alpha)}}\right] .
$$

We derive:

Theorem 2.17. Let $f \in C([m, M]), 0<\alpha<1$. Then

$$
\left\|\left(F_{n}(f)\right)(A)-f(A)\right\| \leqslant(4.1488766)\left[\omega_{1}\left(f, \frac{1}{n^{\alpha}}\right)+2 e^{4}\|f(A)\| e^{-2 n^{(1-\alpha)}}\right] .
$$


We mention:

Theorem $2.18\left(\left[2\right.\right.$, p. 45]). Let $f \in C^{N}([m, M]), N \in \mathbb{N}, 0<\alpha<1$. Then

$$
\begin{aligned}
\left\|F_{n}(f)-f\right\|_{\infty} \leqslant & (4.1488766) \\
& \times\left\{\sum_{j=1}^{N} \frac{\left\|f^{(j)}\right\|_{\infty}}{j !}\left[\frac{1}{n^{\alpha j}}+e^{4}(M-m)^{j} e^{-2 n^{(1-\alpha)}}\right]\right. \\
& \left.+\left[\omega_{1}\left(f^{(N)}, \frac{1}{n^{\alpha}}\right) \frac{1}{n^{\alpha N} N !}+\frac{2 e^{4}\left\|f^{(N)}\right\|_{\infty}(M-m)^{N}}{N !} e^{-2 n^{(1-\alpha)}}\right]\right\} .
\end{aligned}
$$

We derive:

Theorem 2.19. Let $f \in \mathrm{C}^{\mathrm{N}}([\mathrm{m}, \mathrm{M}]), \mathrm{N} \in \mathbb{N}, 0<\alpha<1$. Then

$$
\begin{aligned}
\left\|\left(F_{n}(f)\right)(A)-f(A)\right\| \leqslant & (4.1488766) \\
& \times\left\{\sum_{j=1}^{N} \frac{\left\|f^{(j)}(A)\right\|}{j !}\left[\frac{1}{n^{\alpha j}}+e^{4}(M-m)^{j} e^{-2 n^{(1-\alpha)}}\right]\right. \\
& \left.+\left[\omega_{1}\left(f^{(N)}, \frac{1}{n^{\alpha}}\right) \frac{1}{n^{\alpha N} N !}+\frac{2 e^{4}\left\|f^{(N)}(A)\right\|(M-m)^{N}}{N !} e^{-2 n^{(1-\alpha)}}\right]\right\} .
\end{aligned}
$$

We make:

Definition 2.20 ([3, pp. 332-346]). We consider the (Gauss) error special function

$$
\operatorname{erf}(x)=\frac{2}{\sqrt{\pi}} \int_{0}^{x} e^{-t^{2}} d t, \quad x \in \mathbb{R},
$$

which is a sigmoidal type continuous function and it is a strictly increasing function.

We consider the activation function

$$
\chi(x)=\frac{1}{4}(\operatorname{erf}(x+1)-\operatorname{erf}(x-1)), \quad x \in \mathbb{R} .
$$

Notice $\chi(x)>0$, for all $x \in \mathbb{R}$.

Let $f \in C([m, M]), n \in \mathbb{N}$ such that $n^{1-\alpha} \geqslant 3$, where $0<\alpha<1$.

We consider the positive linear operator

$$
A_{n}(f, x)=\frac{\sum_{k=\lceil n m\rceil}^{\lfloor n M\rfloor} f\left(\frac{k}{n}\right) x(n x-k)}{\sum_{k=\lceil n m\rceil}^{\lfloor n M\rfloor} x(n x-k)}, \quad \forall x \in[m, M]
$$

The operator $A_{n}$ is a neural network operator mapping $C([m, M])$ into itself.

We mention:

Theorem 2.21 ([3, p. 340]). It holds

$$
\left\|A_{n}(f)-f\right\|_{\infty} \leqslant(4.019)\left[\omega_{1}\left(f, \frac{1}{n^{\alpha}}\right)+\frac{\|f\|_{\infty}}{\sqrt{\pi}\left(n^{1-\alpha}-2\right) e^{\left(n^{1-\alpha}-2\right)^{2}}}\right] .
$$


We derive:

Theorem 2.22. It holds

$$
\left\|\left(A_{n}(f)\right)(A)-f(A)\right\| \leqslant(4.019)\left[\omega_{1}\left(f, \frac{1}{n^{\alpha}}\right)+\frac{\|f(A)\|}{\sqrt{\pi}\left(n^{1-\alpha}-2\right) e^{\left(n^{1-\alpha}-2\right)^{2}}}\right] .
$$

We need:

Theorem 2.23 ([3, pp. 345-346]). Let $f \in C^{N}([m, M]), n, N \in \mathbb{N}, n^{1-\alpha} \geqslant 3,0<\alpha<1$. Then

$$
\begin{aligned}
\left\|A_{n}(f)-f\right\|_{\infty} \leqslant & (4.019) \\
& \times\left\{\sum_{j=1}^{N} \frac{\left\|f^{(j)}\right\|_{\infty}}{j !}\left[\frac{1}{n^{\alpha j}}+\frac{(M-m)^{j}}{2 \sqrt{\pi}\left(n^{1-\alpha}-2\right) e^{\left(n^{1-\alpha}-2\right)^{2}}}\right]\right. \\
& \left.+\left[\omega_{1}\left(f^{(N)}, \frac{1}{n^{\alpha}}\right) \frac{1}{n^{\alpha N} N !}+\frac{\left\|f^{(N)}\right\|_{\infty}(M-m)^{N}}{N ! \sqrt{\pi}\left(n^{1-\alpha}-2\right) e^{\left(n^{1-\alpha}-2\right)^{2}}}\right]\right\} .
\end{aligned}
$$

It follows:

Theorem 2.24. Let all as in Theorem 2.23 hold. Then

$$
\begin{aligned}
\left\|\left(A_{n}(f)\right)(A)-f(A)\right\| \leqslant & (4.019) \\
& \times\left\{\sum_{j=1}^{N} \frac{\left\|f^{(j)}(A)\right\|}{j !}\left[\frac{1}{n^{\alpha j}}+\frac{(M-m)^{j}}{2 \sqrt{\pi}\left(n^{1-\alpha}-2\right) e^{\left(n^{1-\alpha}-2\right)^{2}}}\right]\right. \\
& \left.+\left[\omega_{1}\left(f^{(N)}, \frac{1}{n^{\alpha}}\right) \frac{1}{n^{\alpha N} N !}+\frac{\left\|f^{(N)}(A)\right\|(M-m)^{N}}{N ! \sqrt{\pi}\left(n^{1-\alpha}-2\right) e^{\left(n^{1-\alpha}-2\right)^{2}}}\right]\right\} .
\end{aligned}
$$

Conclusion 2.25. Inequalities (2.1), (2.2), (2.3), (2.5), (2.9), (2.6), (2.7), (2.8), (2.9), (2.10) and (2.11), imply $\|(\operatorname{Lf})(A)-f(A)\| \rightarrow 0$, under basic assumptions and imply $\left\|\left(B_{n}(f)\right)(A)-f(A)\right\| \rightarrow 0,\left\|\left(G_{n}(f)\right)(A)-f(A)\right\| \rightarrow$ $0,\left\|\left(F_{n}(f)\right)(A)-f(A)\right\| \rightarrow 0$, and $\left\|\left(A_{n}(f)\right)(A)-f(A)\right\| \rightarrow 0$, as $n \rightarrow \infty$.

The approximations are given quantitatively and with rates via the first modulus of continuity.

\section{References}

[1] G. A. Anastassiou, Quantitative approximations, Chapman \& Hall/CRC, Boca Raton, FL, (2001). 2.1, 2.3, 2.5, 2.7, 2.8

[2] G. A. Anastassiou, Intelligent systems: approximation by artificial neural networks, Intelligent Systems Reference Library, Springer-Verlag, Berlin, (2011). 2.10, 2.11, 2.13, 2.15, 2.16, 2.18

[3] G. A. Anastassiou, Intelligent systems II: complete approximation by neural network operators, Studies in Computational Intelligence, Springer, Cham, (2016). 2.20, 2.21, 2.23

[4] G. A. Anastassiou, Self adjoint operator Korovkin type and polynomial direct approximations with rates, RGMIA Res. Rep. Coll., 19 (2016), 16 pages. 2.7

[5] S. S. Dragomir, Ostrowski's type inequalities for continuous functions of self adjoint operators on Hilbert spaces: a survey of recent results, Ann. Funct. Anal., 2 (2011), 139-205. 1

[6] S. S. Dragomir, Operator inequalities of Ostrowski and trapezoidal type, SpringerBriefs in Mathematics, Springer, New York, (2012). 1

[7] G. Helmberg, Introduction to spectral theory in Hilbert space, North-Holland Series in Applied Mathematics and Mechanics, North-Holland Publishing Co., Amsterdam-London; Wiley Interscience Division John Wiley \& Sons, Inc., New York (1969). 1

[8] C. A. McCarthy, $c_{p}$, Israel J. Math., 5 (1967), 249-271. 1

[9] J. Pečarić, T. Furuta, J. Mićić Hot, Y.-K. Seo, Mond-Pečarić method in operator inequalities, Inequalities for bounded self adjoint operators on a Hilbert space, Monographs in Inequalities, ELEMENT, Zagreb, (2005). 1 